\title{
Schwann-like cell differentiation from rat bone marrow stem cells
}

\author{
Iraj Ragerdi Kashani ${ }^{1}$, Zolikha Golipoor ${ }^{1}$, Mohammad Akbari ${ }^{1}$, Reza Mahmoudi², Shahram Azari ${ }^{3}$, \\ Reza Shirazi ${ }^{1}$, Mohammad Bayat ${ }^{1}$, Soudabeh Ghasemi ${ }^{1}$
}

1Department of Anatomy, School of Medicine, Tehran University of Medical Sciences,
Iran
2Department of Anatomy, School of Medicine, Yasouj University of Medical Sciences,
Iran
${ }^{3}$ National Cell Bank of Iran, Pasteur Institue of Iran

Submitted: 11 November 2009

Accepted: 14 March 2010

Arch Med Sci 2011; 7, 1: 45-52

DOI: 10.5114 /aoms.2011.20603

Copyright (c) 2011 Termedia \& Banach

\begin{abstract}
Introduction: The main purpose of this study was differentiation of bone marrow stem cells (BMSCs) into Schwann-like cells and to determine the intensity of apoptosis in BMSCs during the differentiation process.

Material and methods: Bone marrow stem cells were isolated from the femur of adult rats and the identity of the undifferentiated BMSCs was confirmed by the detection of specific cell surface markers. The BMSCs were differentiated by sequential administration of $\beta$-mercaptoethanol and all-trans-retinoic acid as pre-inducer factors and a mixture of forskolin, basic fibroblast growth factor, platelet-derived growth factor-AA and heregulin-b1 as inducer factors. The immunocytochemical properties of differentiated Schwann-like cells were examined at a specified time point. Reverse transcription-polymerase chain reaction (RT-PCR) was used to investigate the gene expression of the undifferentiated and differentiated BMSCs. Cell apoptosis and viability were assessed with annexin $\mathrm{V}$ and propidium iodide double staining and dimethylthiazol-2-yl-2, 5-diphenyltetrazolium bromide (MTT) assay.

Results: Immunocytochemistry staining and RT-PCR analysis revealed that the induced BMSCs exhibited Schwann cell-specific markers such as S-100, P75 and glial fibrillary acidic protein (GFAP) at the $14^{\text {th }}$ day of differentiation. MTT assay and flow cytometry revealed that of the total BMSCs in the differentiation medium, $40 \%$ to $50 \%$ of the cells died by apoptosis, but the remaining cell population remained strongly attached to the substrate and differentiated.

Conclusions: These findings indicated that BMSCs could differentiate into Schwann-like cells. As a side effect of differentiation an increased cell death rate was noted and our findings indicate that the principle mode of cell death is by apoptosis.
\end{abstract}

Key words: bone marrow, stem cells, apoptosis, Schwann cells.

\section{Introduction}

The transplantation of cultured Schwann cells (SCS) into bio-engineered conduits has been shown to improve nerve regeneration [1]. The ideal "transplantable cell" should be easily accessible, capable of rapid expansion in culture, immunologically inert, and capable of long-term survival and integration in the host tissue [2]. An alternative candidate may be

\author{
Corresponding author: \\ Iraj Ragerdi Kashani \\ Department of Anatomy \\ School of Medicine \\ Tehran University of Medical \\ Sciences \\ Poursina Street \\ Tehran 1417613151, Iran \\ Phone: +98 2164432348 \\ Fax: +98 2166419072 \\ E-mail: iraj.ragerdi@gmail.com
}


bone marrow stem cells (BMSCs) [3]. Recent transplantation studies have suggested that transplantation of undifferentiated BMSCs in rats showed therapeutic benefit after traumatic brain injury $[4,5]$ or spinal cord injury [6]. However, in these post-injury transplantation studies, generally less than $20 \%$ of transplanted cells were immunoreactive for specific antigens [7]. In a different paradigm, in vitro differentiation of BMSCs into cells expressing Schwann cell antigens, followed by in vivo transplantation, was shown to have a regenerative effect on damaged sciatic nerve [8]. This finding suggests that in vitro differentiation of BMSCs into cells of central and peripheral nervous systems, followed by in vivo transplantation, may be a potential therapy for nervous system repair. For cell-based regenerative therapies BMSCs are expanded in vitro and differentiated toward Schwann-like cells by applying culture media contain $\beta$-mercaptoethanol (BME), all-trans-retinoic acid (RA), forskolin, basic fibroblast growth factor, platelet-derived growth factor-AA and heregulin-b1 [9-11]. However, in these in vitro studies, generally less than $50 \%$ of BMSCs were differentiated into Schwann-like cells [11].

Beta-mercaptoethanol [12] and RA [13] are toxic chemicals that were used as pre-inducer factors. Evidence has been presented suggesting that RA is more active in inhibiting proliferation and inducing differentiation in relatively undifferentiated systems, i.e., neuroblastoma and embryonal stem cells [14]. In contrast, in other systems, such as keratinocytes [15] and embryonal lung cells [16], proliferation is stimulated. Moreover, at specific concentrations in culture medium, BME increase BMSCs apoptosis [13]. While the use of specific chemical compounds to induce neurogenic differentiation of BMSCs in culture has been well documented, the potential effects on cell death have not been elucidated [11, 13].

The main purpose of this study was differentiation of BMSCs into Schwann-like cells and to determine the intensity of apoptosis in BMSCs during the differentiation process.

\section{Material and methods}

\section{Isolation and culture of bone marrow stem cells}

About 6- to 8-week-old male Wistar rats of the Albino strain were killed using diethyl ether and the bones were collected under sterile conditions; then all the bones were cut at both ends. The bone marrow from each bone was collected by flushing the bone with $\alpha$ Minimum Essential Medium Eagle ( $\alpha M E M)$ (Sigma, USA) containing $1000 \mathrm{U} / \mathrm{ml}$ Penicillin G (Sigma, USA). After filtering, the cells were centrifuged at $1000 \times \mathrm{g}$ for $5 \mathrm{~min}$. The purified cells were finally dispersed in $\alpha M E M$ with $15 \%$ fetal bovine serum (Sigma, USA) containing $100 \mathrm{U} / \mathrm{ml}$ penicillin and $100 \mu \mathrm{g} / \mathrm{ml}$ streptomycin (Sigma, USA) [17].

\section{Cell culture and expansion}

The isolated BMSCs were plated in T75 tissue culture flasks containing appropriate stem cell medium (Sigma, USA) at a density of $10 \times 10^{5}$ cells per flask. The flasks were maintained in a tissue culture incubator at $37^{\circ} \mathrm{C}$ and $5 \%$ carbon dioxide. The medium was replaced every third day afterwards. Cell viability was confirmed by continued cell division and the cells were subcultured using $3 \mathrm{ml}$ of trypsin/EDTA (Sigma, USA) when the flasks reached $90 \%$ confluence [17].

\section{Primary culture of Schwann cells}

Primary Schwann cells were obtained by methods similar to those first described by Brockes et al. [18].

\section{Flow cytometry}

Rat BMSCs within 3-5 passages after the initial plating of the primary culture were harvested by trypsinization, and then the cells were fixed in neutralized $2 \%$ paraformaldehyde solution (Sigma, USA) for $30 \mathrm{~min}$. The fixed cells were washed twice with phosphate buffer saline (PBS) (Sigma, USA) and incubated with antibodies to the following antigens: CD31, CD45, CD90 and CD44 (all from Chemicon, CA) for 30 min. Primary antibodies were directly conjugated with FITC. Flow cytometry was performed with a FACScan flow cytometer (Becton Dickinson, CA) [19].

\section{BMSCs multilineage differentiation potential}

The adipogenic and osteogenic differentiation capacity of BMSCs was confirmed according to the previously published protocols [11].

\section{Differentiation of BMSCs to SC-like cells}

After subculturing at the concentration of $10 \times 10^{5}$ cells $/ \mathrm{cm}^{2}$, BMSCs were incubated in $\alpha M E M$ containing $1 \mathrm{mM}$ BME without serum for $24 \mathrm{~h}$. The culture medium was then replaced with $\alpha M E M$ containing 10\% FBS and $35 \mathrm{ng} / \mathrm{ml} \mathrm{RA}$ (Sigma, USA). Three days later, cells were finally transferred to inducer medium containing aMEM, 10\% FBS and trophic factors of $5 \mu \mathrm{M}$ FSK (Calbiochem, CA), $10 \mathrm{ng} / \mathrm{ml}$ bFGF (Peprotech, UK), 5 ng/ml PDGF (Peprotech, UK), and 200 ng/ml HRG (R\&D systems, USA) and cultured for 10 days [9].

\section{Immunostaining of cultured cells}

Differentiated BMSCs cultured on chamber slides (Lab-Tek, Denmark) were fixed in $4 \%(\mathrm{w} / \mathrm{v})$ 
paraformaldehyde at $4^{\circ} \mathrm{C}$ for $20 \mathrm{~min}$. Cell nuclei were labelled with 6 diamidino-2-phenylindole dihydrochloride (DAPI, Sigma-Aldrich) $(1 \mu \mathrm{g} / \mathrm{ml})$ for $60 \mathrm{~min}$ at room temperature. Cells were then incubated overnight at $4^{\circ} \mathrm{C}$ with primary antibodies to S100 (rabbit polyclonal; 1 : 200; Dako, Denmark). The following day, slides were incubated for 2 hour with fluorescein isothiocyanate (FITC)-conjugated secondary antibody (horse anti-mouse or goat antirabbit; 1 : 100; Vector Labs., USA). Slides were examined under a fluorescence microscope (Olympus BX60). Cultures of Schwann cells were similarly stained as positive controls according to the antibodies used [11].

RNA extraction and reverse transcription polymerase chain reaction (RT-PCR) analysis of gene expression

After the pre-induction and the induction periods, the total RNA was extracted from the induced BMSCs, the non-induced cells and Schwann cells using RNX plus (CinnaGen) and was stored at $-70^{\circ} \mathrm{C}$. The CDNA synthesis was carried out from $5 \mu \mathrm{g}$ total RNA using Fermentas kit (Fermentas) according to the manufacturer's instructions. The cDNA was amplified (the primers of the genes in this study are the following components: $0.2 \mathrm{pM}$ of each primer, $0.3 \mathrm{mMdNTP}, 1.5 \mathrm{mM} \mathrm{MgCl2}, 1$ Utaq DNA polymerase and $1 \times$ PCR buffer (Fermentas). The PCR reactions were conducted in a programmable thermocycler (Bio-Rad) with the following temperature profile: $94^{\circ} \mathrm{C}$ for $5 \mathrm{~min}, 35$ cycles at $94^{\circ} \mathrm{C}$ for $45 \mathrm{~s}, 55^{\circ} \mathrm{C}$ for $45 \mathrm{~s}$ and $72^{\circ} \mathrm{C}$ for $45 \mathrm{~s}$, and a final extension at $72^{\circ} \mathrm{C}$ for $10 \mathrm{~min} ; 10 \mu \mathrm{g}$ of the PCR product was separated, run on a $1.5 \%$ agarose gel and stained with ethidium bromide [10].

\section{Cytotoxicity assay}

The BMSCs were incubated with $\beta$-mercaptoethanol, all-trans-retinoic acid and inducer medium respectively. Prior to measuring viability, treatment media were removed and replaced with $100 \mu \mathrm{l}$ fresh 10\% FBS/DMEM medium and $100 \mu \mathrm{l}$ 3-(4,5-dimethylthiazol-2-yl)-2, 5-diphenyltetrazolium bromide (MTT) (Sigma, USA). Cells were then returned listed in Table I). The $25 \mu$ I PCR product contained

to the incubator for an additional $4 \mathrm{~h}$ before $50 \mu \mathrm{l}$ of $10 \%$ SDS was added to stop the reaction. Optical density (OD) at $570 \mathrm{~nm}$ was read on a microplate reader to indirectly reflect the number of viable cells. The inhibition rate (IR, IR = [1-ODtest/ODcontrol] $\times 100$ ) was used to evaluate the cytotoxicity of preinducer and inducer media [20].

\section{Annexin V/PI assays}

To confirm apoptosis induction, annexin V, and propidium iodide $(\mathrm{PI})$ double staining was performed by using the Annexin V-FITC Apoptosis Detection Kit as described by the manufacturer (Cat. No. 556420; BD Biosciences Pharmingen, San Jose, CA). The cells stained with annexin V-FITC were analysed with a flow cytometer (FACScan; Becton Dickinson, Mountain View, CA) using Cell Quest Software (BD Biosciences), which was also used to determine the percentage of apoptotic and/or necrotic cells [21].

\section{Statistical analysis}

The data were analysed with one-way ANOVA and Tukey's test.

\section{Results}

\section{Rat BMSCs characterization}

The BMSCs appeared as a monolayer of large, fibroblast-like flattened cells (Figure 1. A) which when treated with appropriate induction media

Table I. Primer sequences used for RT-PCR

\begin{tabular}{|lcc|}
\hline Gene & & \multicolumn{2}{c|}{ Primers } \\
\hline P75 & Forward & 5'-CATCTCTGTGGACAGCCAGA-3' \\
\cline { 2 - 3 } & Reverse & 5'-CTCTACCTCCTCACGCTTGG-3' \\
\hline S100 & Forward & 5'-ATAGCACCTCCGTTGGACAG-3' \\
\cline { 2 - 3 } & Reverse & 5'-TCGTTTGCACAGAGGACAAG-3' \\
\hline GFAP & Forward & 5'-GGTGTGGAGTGCCTTCGTAT-3' \\
\cline { 2 - 3 } & Reverse & 5'-TACGATGTCCTGGGAAAAGG-3' \\
\hline Beta actin & Forward & 5'-CTGGCACCCAGCACAATG-3' \\
\cline { 2 - 3 } & Reverse & 5'-AGCGAGGCCAGGATGGA-3' \\
\hline
\end{tabular}
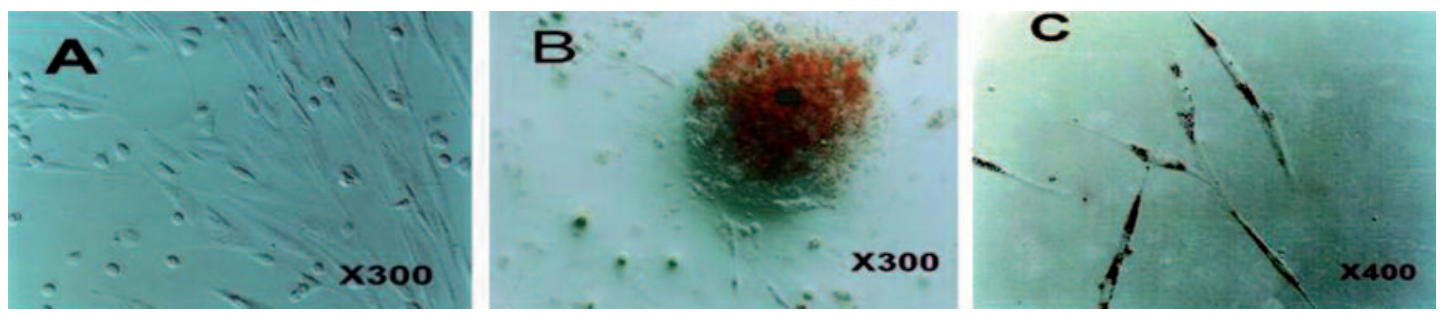

Figure 1. Undifferentiated BMSCs, under phase contrast microscopy, display a flattened fibroblast-like morphology (A). Alizarin Red staining of mineralised bone tissue (B) and Oil Red O positive intracellular lipid droplets (C) indicate that these cells can differentiate to osteoblasts and adipocytes at ADSC 

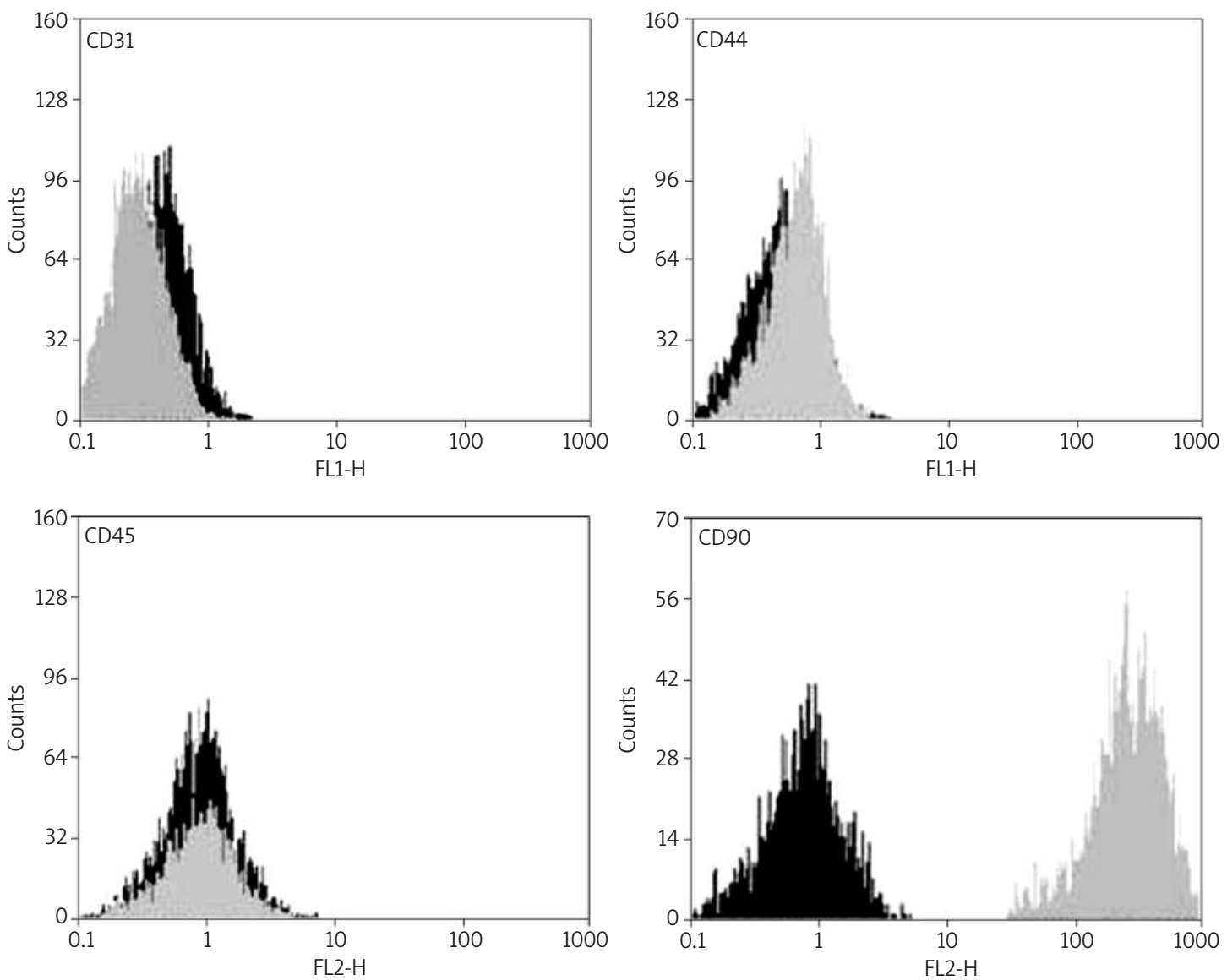

Figure 2. Flow cytometric analysis of rat BMSCs. Rat BMSCs within 3-5 passages were harvested and specific cell surface antigens were detected. Cells stained with FITC-conjugated non-specific IgG were examined as a control (FITC). Flow cytometric analysis shows that rat BMSCs do not express CD31 and CD45, but express CD90 and CD44

where able to adopt an osteogenic (Figure 1. B) or adipogenic (Figure 1. C) phenotype.

Flow cytometry analysis of rat BMSCs within 3-5 passages showed that rat BMSCs were CD90 and CD44 positive, but CD31 and CD45 negative (Figure 2).

\section{Immunocytochemistry of BMSCs after induction}

We detected the expression of SC marker S-100 to evaluate the nature of SC-like cells. Almost all the differentiated BMSCs and Schwann cells were also positive to the S-100 antibody (Figure 3). After 14 days of induction, expression of S-100 protein was demonstrated in the cytoplasm of SC-like cells.

\section{RT-PCR results}

The presence of the glial cell marker gene transcripts was detected by RT-PCR methodology (Figure 4). Before differentiation induction, BMSCS were shown to be negative for S100, P75 and GFAP. After 14 days of induction, BMSCs expressed the transcripts for S100, P75 and GFAP compared to the undifferentiated BMSCS. The intensity of S100 expression was higher than that of GFAP and similar to the Schwann cell group. RT-PCR amplification efficacy of the mRNA was confirmed by amplification of the constitutively expressed beta actin housekeeping gene (Figure 4).

\section{Cytotoxicity assay}

To study the cytotoxicity effect of BME, RA and inducer medium on BMSCs during differentiation into Schwann-like cells, we evaluated the viability of BMSCs by MTT assay. This assay usually shows high correlation with the number of living cells, cell proliferation and release of mitochondrial matrix enzymes. The BME, RA and inducer medium significantly reduced BMSCs viability as assessed by MTT assay (Figure 5).

\section{Quantification of apoptosis by flow cytometry}

By staining cells with annexin V-FITC and PI, FACS was used to distinguish and quantitatively determine the percentage of viable cells (lower 

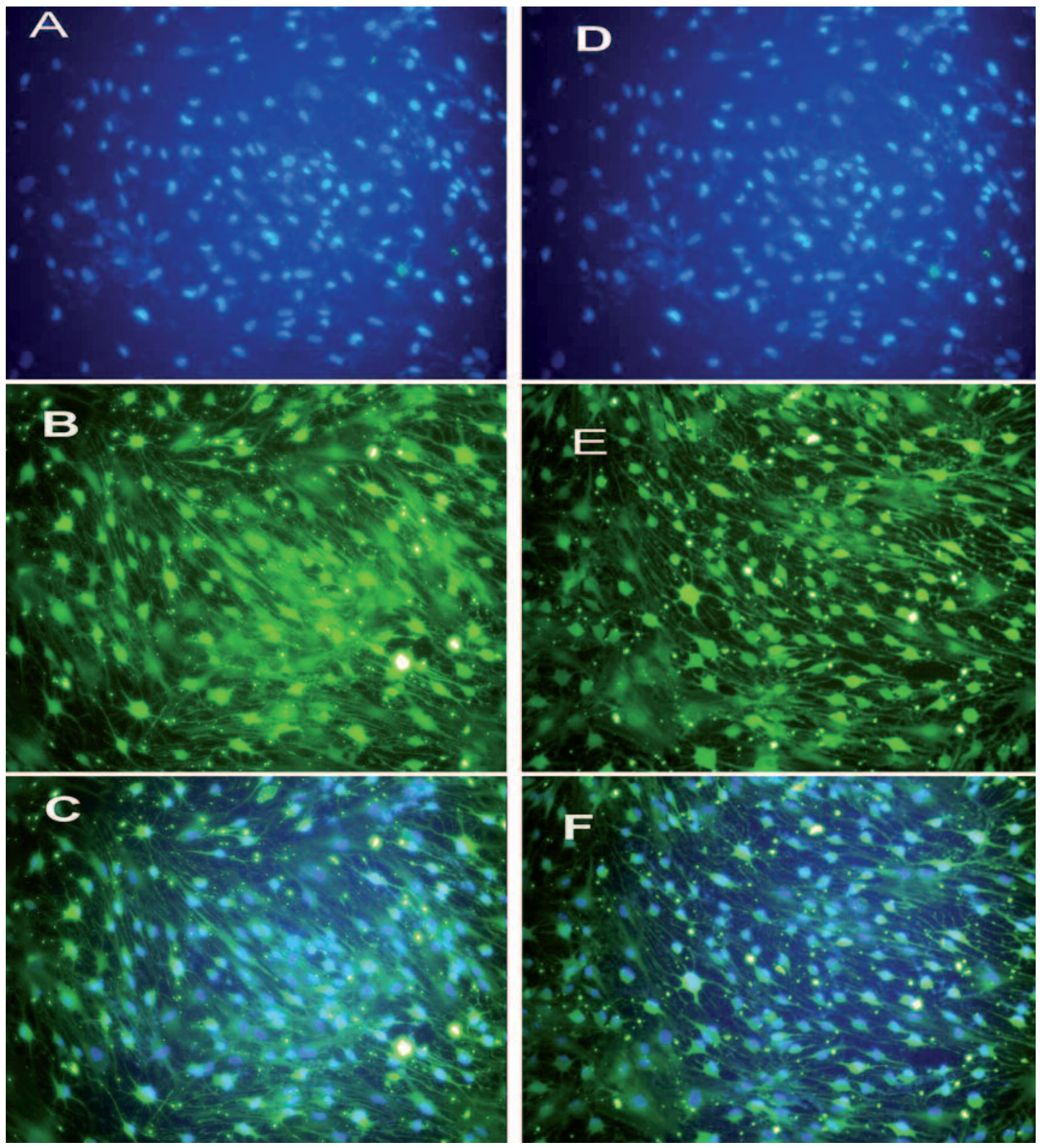

Figure 3. Immunocytochemistry of S100 in differentiated BMSCs (A-C) and rat Schwann cell-line (D-F). After the induction, differentiated BMSCs became positive for S100 antibody. Rat Schwann cell-line was used as a positive control. DAPI staining (A, D); S100 antibody (B, E); merge (C, F)

left quadrant), apoptotic cells (lower right quadrant), late apoptotic necrotic cells (upper right quadrant) and necrotic cells (upper left quadrant) after sequential administration of $\beta$-mercaptoethanol, all-trans-retinoic acid and a mixture of FSK, bFGF, PDGF and HRG was used to induce differentiation of BMSCs into Schwannlike cells (Figure 6. A). The proportions of viable cells, early apoptotic cells, late apoptotic/necrotic and necrotic cells for the control group were $94.34 \%, 2.34 \%, 1.62 \%$ and $1.7 \%$ respectively. For cells treated with $\beta$-mercaptoethanol, the proportions were $78 \%, 14 \%, 6 \%$ and $2 \%$ respec- tively. For cells treated with all-trans-retinoic acid, the proportions were $75.84 \%, 19.31 \%, 3.53 \%$ and $1.32 \%$ respectively. For cells treated with inducer medium proportions were $80.84 \%, 13.93 \%, 5 \%$ and $0.23 \%$ (Figure 6. B).

\section{Discussion}

Over the past decade, stem cells from adult bone marrow have been exploited as therapeutic vectors in the treatment of a wide variety of diseases [4, 5]. This study clearly shows the plasticity of BMSCS by their differentiation into Schwann-like cells 


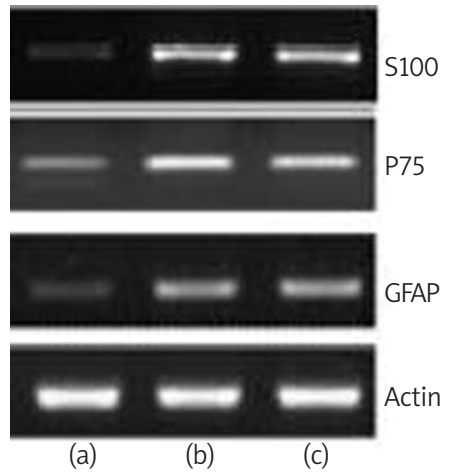

Figure 4. Transdifferentiated BMSCs were assayed by RT-PCR for S-100, P75 and GFAP mRNA. Undifferentiated BMSCs (a), transdifferentiated BMSCs (b) and rat Schwann cell-line was used as a positive control (c)

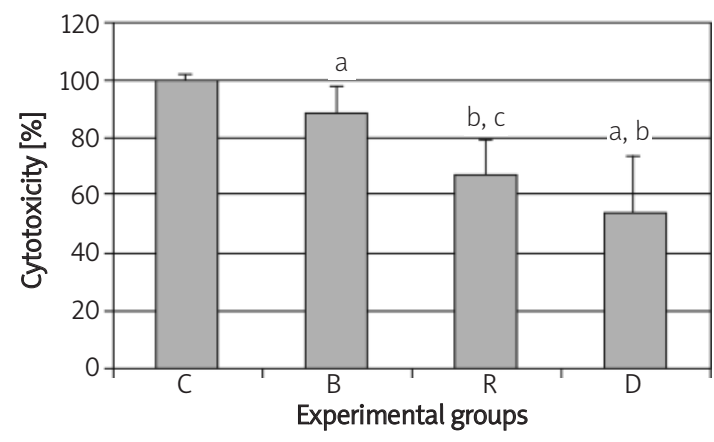

Figure 5. Histogram shows the cytotoxicity of $\beta$-mercaptoethanol, all-trans-retinoic acid and inducer medium on BMSCs identified by MTT assay. $C, B, R$ and $D$ are non-treated cells (control), preinduced by $\beta$-mercaptoethanol, pre-induced by alltrans-retinoic acid and induced by differentiated medium respectively

$a$ - significant difference with control group, $b$ - significant difference with $\beta$-mercaptoethanol group, $c$ - significant difference with all-trans-retinoic acid group, $d$-significant difference with induction medium group $(P<0.05)$

with the typical spindle-shaped Schwann cell morphology. In this study we have confirmed that the BMSCs express characteristic mesenchymal stem cell (MSC) surface markers (CD44 and CD90 positive, but CD31 and CD45 negative) and also demonstrated the multilineage potential of BMSCs [13]. Clear evidence is provided to show that rat BMSCs have the ability to differentiate along a glial lineage and express cell markers (S100, P75 and GFAP) which are typical for glial cells including Schwann cells [3-8].

On the basis of our results, the sequential administration of various factors, BME, RA, followed by a mixture of FSK, bFGF, PDGF and HRG, effectively induces the differentiation of BMSCs into Schwann-like cells. The phenotype of the differentiated BMSCs resembled that of Schwann cells.
The previous reports showed that the addition of BME to cultured BMSCs induced the formation of neurite-like processes [14]. RA is a morphogenic factor, and was reported to induce differentiation of neural stem cells into nerve cells [15]. Therefore, BME and RA are presumed to work as triggering factors, inducing changes in the morphological and transcriptional characteristics of BMSCs.

Previous studies reported that bFGF functions as a mitogen for BMSCs, and that MAP kinases are activated by PDGF and bFGF in MSCs [16]. HRG, a subtype of neuregulin, instructively influences the decision of cell fates and was reported to induce neural crest cells to develop selectively into Schwann cells [17].

FSK increases the level of intracellular cyclic adenosine monophosphate (CAMP), which increases mitogenic responses at an early time [18]. As CAMP elevation is reported to enhance the responsiveness of cells to trophic factors [19], FSK together with bFGF, PDGF, and HRG could have a synergistic effect in enhancement of these factors to BMSCs [20].

MTT assay and flow cytometry analysis for annexin $\mathrm{V}$ and $\mathrm{Pl}$ indicated that during the chemical induction process there was an increase in cell death. Putative early apoptotic cells increased after exposure to BME and RA.

Measures of cell death during this specific induction process are indicative of toxicity of the chemical compounds used. Here we show that almost $50 \%$ of cells die within 48 h of chemical induction, in agreement with other studies [8, 10, 21].

Our results indicate that RA exerts two apparently opposite effects on cultured ADSCs, i.e., a differentiating effect and induction of cell death. Flow cytometry analysis for annexin V and PI indicated that putative early apoptotic cells increased after exposure to RA. These data agreed with previous reports where all-trans-retinoic acid produced similar effects in cancers [22] and stroke [23].

The mechanism of the apoptotic effects of $\beta$-mercaptoethanol and all-trans-retinoic acid is unknown and there are several pathways that need to be investigated.

Oliva et al. reported that antiproliferative activity of retinoic acid is associated with (and probably due to) the up-regulation of two pivotal cdk inhibitors, and subsequent cdk2 activity decrease and retinoblastoma protein ( $\mathrm{pRb}$ ) hypophosphorylation [24].

$\mathrm{pRb}$ is a tumour suppressor protein that is dysfunctional in many types of cancer [25]. In the hypophosphorylated state, pRb is active and carries out its role as a tumour suppressor by inhibiting cell cycle progression [26].

However, there is evidence indicating that either direct contact with axons or survival factors secreted by neurons are necessary for the survival 
(a)

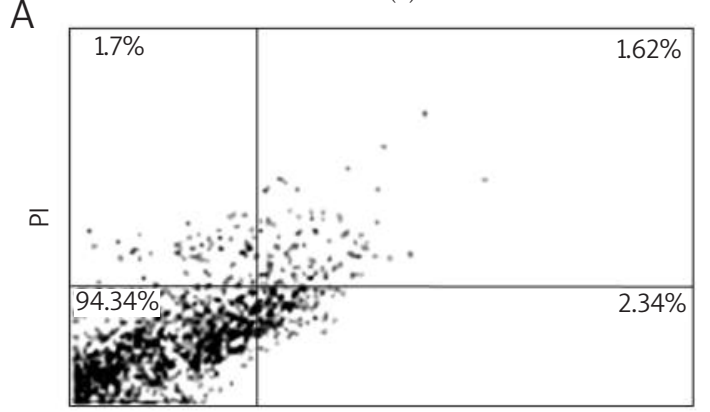

(c)

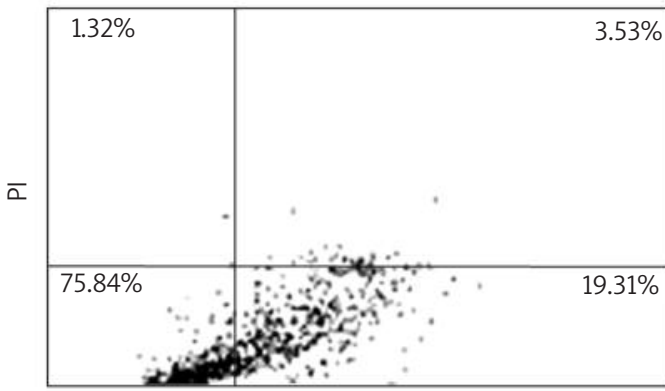

B

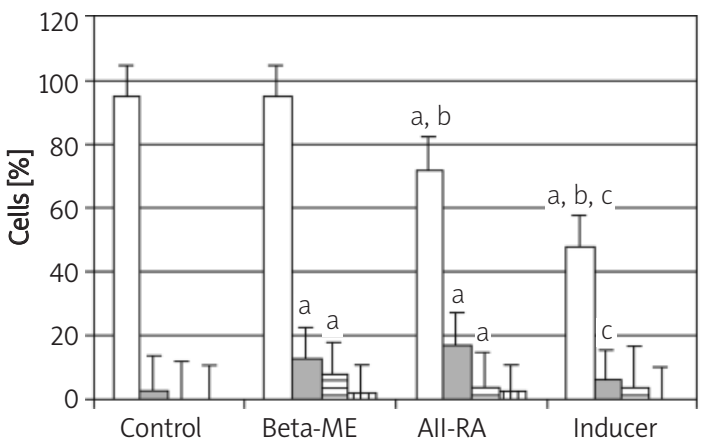

and development of SC precursors as well as of mature Schwann cell [8].

In conclusion, these findings indicated that BMSCs could differentiate into Schwann-like cells in terms of morphology and phenotype. As a side effect of differentiation an increased cell death rate was noted and our findings indicate that the principle mode of cell death is by apoptosis.

Hence, a considerable amount of BMSCs would die during differentiation into Schwann-like cells, depending on the method used. This may compromise the optimal outcome of the engraftment and subsequently nerve regeneration and repair.

\section{Acknowledgment}

This study was supported by Tehran University of Medical Sciences and Health Services, Tehran, Iran (grant No. 8382-30-01-88). (b)

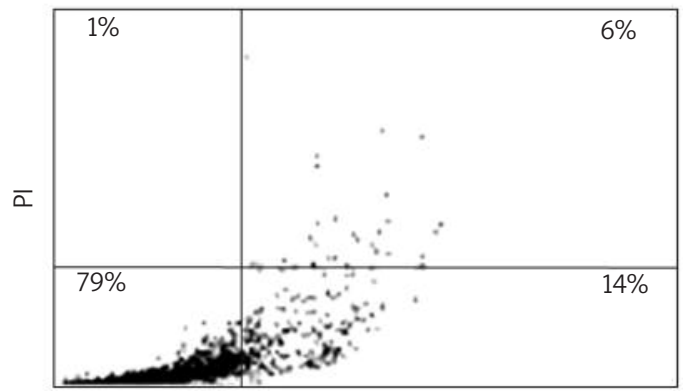

(d)

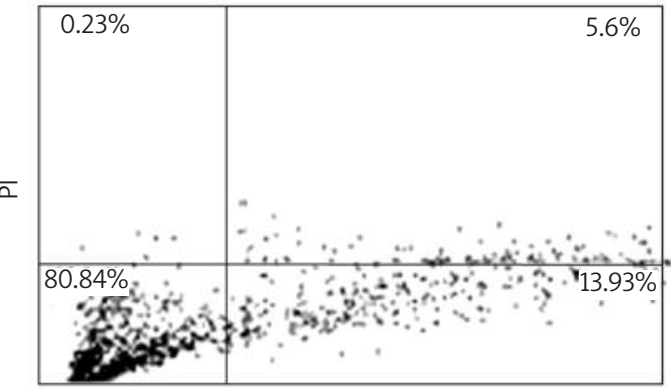

Figure 6. (A) Analysis of BMSC apoptosis before and after differentiation into Schwann-like cells by flow cytometry. FACS analysis after staining with annexin V/PI. Three distinct cell distribution patterns are visible: normal viable cells (lower left quadrant), apoptotic cells (lower right quadrant), late apoptotic or necrotic cells (upper right quadrant) and necrotic cells (upper left quadrant). (a) Non-treated cells (control), (b) induced by $\beta$-mercaptoethanol, (c) induced by all-transretinoic acid, (d) induced by differentiated medium. (B) Histogram shows the percentage of rat BMSCs' apoptosis before and after differentiation into Schwann-like cell phenotype as identified by annexin V/PI flow cytometry $a$ - significant difference with control group, $b$ - significant difference with $\beta$-mercaptoethanol group, $c$ - significant difference with all-transretinoic acid group, $d$ - significant difference with induction medium group $(P<0.05)$

\section{References}

1. Mosahebi A, Woodward B, Wiberg M, Martin R, Terenghi G. Retroviral labeling of Schwann cells: in vitro characterization and in vivo transplantation to improve peripheral nerve regeneration. Glia 2001; 34: 8-17.

2. Azizi SA, Stokes D, Augelli BJ, DiGirolamo C, Prockop DJ. Engraftment and migration of human bone marrow stromal cells implanted in the brains of albino rats similarities to astrocyte grafts. Proc Natl Acad Sci U S A 1998; 95: 3908-13.

3. Brohlin M, Mahay D, Novikov LN, Terenghi G, Wiberg M, Shawcross SG, Novikova LN. Characterisation of human mesenchymal stem cells following differentiation into Schwann cell-like cells. Neurosci Res 2009; 64: 41-9.

4. Mahay D, Terenghi G, Shawcross SG. Schwann cell mediated trophic effects by differentiated mesenchymal stem cells. Exp Cell Res 2008; 314: 2692-701.

5. Shimizu S, Kitada M, Ishikawa H, Itokazu Y, Wakao S, Dezawa $M$. Peripheral nerve regeneration by the in vitro differentiated-human bone marrow stromal cells with Schwann cell property. Biochem Biophys Res Commun 2007; 359: 915-20. 
6. Lin W, Chen X, Wang X, Liu J, Gu X. Adult rat bone marrow stromal cells differentiate into Schwann cell-like cells in vitro. In Vitro Cell Dev Biol Anim 2008; 44: 31-40.

7. Caddick J, Kingham PJ, Gardiner NJ, Wiberg M, Terenghi G. Phenotypic and functional characteristics of mesenchymal stem cells differentiated along a Schwann cell lineage. Glia 2006; 54: 840-9.

8. Keilhoff G, Stang F, Goihl A, Wolf G, Fansa H. Transdifferentiated mesenchymal stem cells as alternative therapy in supporting nerve regeneration and myelination. Cell Mol Neurobiol 2006; 26: 1235-52.

9. Kalbermatten DF, Kingham PJ, Mahay D, et al. Fibrin matrix for suspension of regenerative cells in an artificial nerve conduit. J Plast Reconstr Aesthet Surg 2008; 61: 669-75.

10. Barnabé GF, Schwindt TT, Calcagnotto ME, et al. Chemically-induced RAT mesenchymal stem cells adopt molecular properties of neuronal-like cells but do not have basic neuronal functional properties. PLoS One 2009; 4: e5222.

11. Zaminy A, Ragerdi Kashani I, Barbarestani M, Hedayatpour A, Mahmoudi R, Farzaneh Nejad A. Osteogenic differentiation of rat mesenchymal stem cells from adipose tissue in comparison with bone marrow mesenchymal stem cells: melatonin as a differentiation factor. Iran Biomed J 2008; 12: 133-41.

12. Brockes JP, Fields KL, Raff MC. Studies on cultured rat Schwann cells. Establishment of purified populations from cultures of peripheral nerve. Brain Res 1979; 165: 105-18.

13. Nadri S, Soleimani M, Hosseni RH, Massumi M, Atashi A, Izadpanah R. An efficient method for isolation of murine bone marrow mesenchymal stem cells. Int J Dev Biol 2007; 51: 723-9.

14. Woodbury D, Schwarz EJ, Prockop DJ, Black IB. Adult rat and human bone marrow stromal cells differentiate into neurons. J Neurosci Res 2000; 61: 364-70.

15. Kingham PJ, Kalbermatten DF, Mahay D, Armstrong SJ, Wiberg M, Terenghi G. Adipose-derived stem cells differentiate into a Schwann cell phenotype and promote neurite outgrowth in vitro. Exp Neurol 2007; 207: 267-74.

16. Chaudhary LR, Avioli LV. Activation of extracellular signalregulated kinases 1 and 2 (ERK1 and ERK2) by FGF2 and PDGF-BB in normal human osteoblastic and bone marrow stromal cells: differences in mobility and in-gel renaturation of ERK1 in human, rat, and mouse osteoblastic cells. Biochem Biophys Res Commun 1997; 238: 134-9.

17. Morrison SJ, White PM, Zock C, Anderson DJ. Prospective identification, isolation by flow cytometry, and in vivo selfrenewal of multipotent mammalian neural crest stem cells. Cell 1999; 96: 737-49.

18. Shah NM, Groves AK, Anderson DJ. Alternative neural crest cell fates are instructively promoted by TGFbeta superfamily members. Cell 1996; 85: 331-43.

19. Kim HA, Ratner N, Roberts TM, Stiles CD. Schwann cell proliferative responses to CAMP and $\mathrm{NF} 1$ are mediated by cyclin D1. J Neurosci 2001; 21: 1110-6.

20. Dezawa M, Takahashi I, Esaki M, Takano M, Sawada H. Sciatic nerve regeneration in rats induced by transplantation of in vitro differentiated bone-marrow stromal cells. Eur J Neurosci 2001; 14: 1771-6.

21. Rismanchi N, Floyd CL, Berman RF, Lyeth BG. Cell death and long-term maintenance of neuron-like state after differentiation of rat bone marrow stromal cells: a comparison of protocols. Brain Res 2003; 991: 46-55.

22. Cunningham TJ, Jing H, Akerblom I, Morgan R, Fisher TS, Neveu M. Identification of the human cDNA for new survival/evasion peptide (DSEP): studies in vitro and in vivo of overexpression by neural cells. Exp Neurol 2002; 177: 32-9.

23. Harvey BK, Shen H, Chen GJ, Yoshida Y, Wang Y. Midkine and retinoic acid reduce cerebral infarction induced by middle cerebral artery ligation in rats. Neurosci Lett 2004; 369: 138-41.

24. Oliva A, Borriello A, Zeppetelli S, et al. Retinoic acid inhibits the growth of bone marrow mesenchymal stem cells and induces p27Kip1 and p16INK4A up-regulation. Mol Cell Biochem 2003; 247: 55-60.

25. Murphree AL, Benedict WF. Retinoblastoma: clues to human oncogenesis. Science 1984; 223: 1028-33.

26. Vietri M, Bianchi M, Ludlow JW, Mittnacht S, Villa-Moruzzi E. Direct interaction between the catalytic subunit of Protein Phosphatase 1 and pRb. Cancer Cell Int 2006; 6: 3. 